\title{
MicroRNA-Let-7f reduces the vasculogenic mimicry of human glioma cells by regulating periostin-dependent migration
}

\author{
HAO XUE ${ }^{1,2^{*}}$, XIAO GAO $^{1 *}$, SHUGANG XU ${ }^{1,3^{*}}$, JINSEN ZHANG $^{1,2}$, XING GUO $^{1,2}$, SHAOFENG YAN $^{1}$, \\ TONG LI ${ }^{1}$, XIAOFAN GUO ${ }^{1}$, QINGLIN LIU ${ }^{1}$ and GANG LI ${ }^{1,2}$ \\ ${ }^{1}$ Department of Neurosurgery, Qilu Hospital of Shandong University; ${ }^{2}$ Brain Science Research Institute, \\ Shandong University, Jinan, Shandong 250012; ${ }^{3}$ Department of Neurosurgery, \\ Dezhou People's Hospital, Dezhou, Shandong 253014, P.R. China
}

Received October 28, 2015; Accepted December 5, 2015

DOI: $10.3892 / o r .2016 .4548$

\begin{abstract}
The present study was the first to examine the effect of microRNA-Let-7f (miR-Let-7f) inhibiting vasculogenic mimicry (VM) of human glioma cells. The postoperative survival time was significantly poor in VM-positive glioma patients compared with those without VM. Thus, it is reasonable to postulate that miR-Let-7f functions as a potent tumor suppressor by inhibiting glioma VM. However, the molecular mechanisms involved remain poorly clarified. Our preliminary studies revealed that miR-Let-7f suppressed VM by disturbing periostin (POSTN)-induced migration of glioma cells. Our results clearly demonstrated that inhibiting the pro-migratory function of POSTN by the overexpression of miR-Let-7f significantly reduced the formation of VM. Our findings suggest that miR-Let-7f may serve as a potential complementary therapeutic target in the anti-angiogenesis treatment of gliomas via suppressing VM.
\end{abstract}

\section{Introduction}

Glioma, which is the most malignant tumor type, accounts for more than $70 \%$ of all brain tumors (1). The most common subtype is glioblastoma multiforme (GBM), with age-adjusted incidence rate ranging from 0.59 to $3.69 / 100,000$ persons (2). The biological properties of GBM primarily include high mortality and recurrence rates, uncontrollable invasiveness, strong angiogenesis (3) and widespread hypoxia (4). Tumor

Correspondence to: Dr Qinglin Liu or Professor Gang Li, Department of Neurosurgery, Qilu Hospital of Shandong University China, 107 Wenhua Western Road, Jinan, Shandong 250012, P.R. China

E-mail: bodystrong@126.com

E-mail: ligangqiluhospital@163.com

\section{${ }^{*}$ Contributed equally}

Key words: microRNA, glioma, vasculogenic mimicry, hypoxia, prognosis, migration angiogenesis is an independent prognostic factor associated with poor survival (4). In fact, there is increasing evidence that hypoxia activates angiogenesis (5), metastasis (6) and many other cellular processes in tumors. Although multitude of mechanisms have been proposed to elucidate the hypoxia-induced angiogenesis of tumor cells, more research is warranted to determine the role of a new way of angiogenesis in mediating the effects of hypoxia.

Vasculogenic mimicry (VM) is a new tumor vascular pattern different from angiogenesis (7). It describes a specific capacity of aggressive tumor cells to form vessel-like networks without vascular endothelial cells that provide adequate blood supply for tumor growth $(7,8)$. A variety of molecular mechanisms and signal pathways participate in VM induction (8). Additionally, tumor stem cells are also shown to be implicated in VM formation (9). As a unique vessel formation manner, $\mathrm{VM}$ is associated with tumor invasion and poor patient prognosis in various tumors (10). Due to its important effects on tumor progression, VM-related target molecules and strategies are being studied for anticancer treatment (8). However, the specific molecular mechanisms of VM in glioma are still unclear.

miRNAs are a class of endogenous small non-coding RNAs that have been identified as negative regulators of gene expression at the post-transcriptional level $(11,12)$. These small molecules are incorporated into the RNA-induced silencing complex and bind to the seed sequence in the 3'-untranslated regions (3'-UTRs) of their target mRNAs to silence gene translation via mRNA degradation, translational repression and/or miRNA-mediated mRNA decay (12). Therefore, additional research is warranted to determine the important roles of numerous miRNAs in diverse tumor-related cellular processes, such as proliferation (13), angiogenesis (14) and metastasis (15). In gliomas, various tumor-promoting and tumor-suppressing miRNAs (16-19) have been identified. However, there are only few miRNAs targets discovered regulating tumor VM (20-22). Our observation suggest that miRNAs may be important for the tumor VM and provide new insights into understanding the molecular mechanism underlying tumor progression.

The value and novelty of the present study is to indicate, for the first time in human glioma that microRNA-Let-7f 
(miR-Let-7f) is a VM-negative regulator by targeting the periostin (POSTN)-induced migration. Firstly, we demonstrated $\mathrm{VM}$ as an indicator of poor prognosis by testing its positive rate in human glioma tumor samples. Then, we investigated the expression of miR-Let-7f in human glioma tumor samples, and we found that miR-Let-7f functions as a clear VM suppressor. Further study using the glioma cell line A172 revealed that miR-Let-7f knockdown drastically enhanced the VM forming capacity of glioma cells and the miR-Let-7f overexpressed glioma cells almost completely lost the VM forming ability. Using specific small interfering RNA (siRNA), POSTN was considered as the potential intermediary anti-VM molecule of miR-Let-7f. These findings suggest an unexpected fundamental tumor-suppressive role for miR-Let-7f in glioma due to its anti-VM effect.

\section{Materials and methods}

Tissue samples and cell culture. The human glioma cell line A172 was purchased from the Chinese Academy of Sciences Cell Bank. All cells were cultured in Dulbecco's modified Eagle's medium (DMEM) supplemented with $10 \%$ fetal bovine serum (FBS) and maintained at $37^{\circ} \mathrm{C}$ with $5 \% \mathrm{CO}_{2}$ in a humidified chamber. Hypoxic conditions were induced by incubating the cells in a modular incubator chamber with a gas mixture containing $1 \% \mathrm{O}_{2}, 5 \% \mathrm{CO}_{2}$ and $94 \% \mathrm{~N}_{2}$ at $37^{\circ} \mathrm{C}$. Forty-five human glioma tissues including 4 World Health Organization (WHO) grade I tumors, 8 grade II tumors, 13 grade III tumors and 20 grade IV tumors, and 2 normal brain tissues of decompression operation were obtained from the Department of Neurosurgery of Qilu Hospital of Shandong University. The glioma specimens were verified and classified according to the WHO classification standard of tumors by two experienced clinical pathologists. The present study was approved by the Institutional Review Board of Shandong University. Written informed consent was obtained from all patients, and the hospital Ethics Committee approved the experiments.

Cell transfection. Mature miR-Let-7f mimics, the scrambled mimic control, the miR-Let-7f inhibitor, the scrambled inhibitor control, the miR-584-5p mimic and POSTN siRNA were designed and synthesized by RiboBio. Cell transfections and co-transfections were performed using Lipofectamine 2000 when the cells reached $70 \%$ confluency according to the manufacturer's instructions. Untransfected cells were used as the blank control, while cells transfected with the scrambled oligos were used as the negative controls. The transfection efficiency was verified by quantitative real-time PCR. Forty-eight hours after transfection, the glioma cells were harvested for subsequent experiments.

Cell viability assay. A172 cells were seeded into 96-well culture plates at a density of 3,000 cells/well. Cell proliferation was analyzed at 24, 48, 72 and $96 \mathrm{~h}$ after transfection using a Cell Counting Kit-8 (CCK-8). A volume of $10 \mu \mathrm{l}$ of CCK-8 solution was added to each well, and the cells were incubated for another $1 \mathrm{~h}$ in a humidified incubator at $37^{\circ} \mathrm{C}$. Optical density was measured at $450 \mathrm{~nm}$ using a microplate reader. Each assay was performed in triplicate.
Wound healing and cell migration assays. The transfected cells $\left(1 \times 10^{5}\right)$ were seeded in 6-well plates, incubated overnight, and at $90 \%$ confluency, the cell monolayer was scratched with a sterile pipette tip. The scratched plates were cultured in DMEM containing 1\% FBS. Images were captured at 0 and $12 \mathrm{~h}$ along the scrape line under a microscope. Cell migration assays were performed using Transwell chambers $(8-\mu \mathrm{m}$; Corning). In total, $5 \times 10^{4}$ transfected cells in FBS-free medium were seeded in the upper chamber. Medium containing $10 \%$ FBS was added to the lower chamber. After $12 \mathrm{~h}$, the cells that did not migrate or invade were removed using cotton buds. The cells migrating on the lower surface were fixed and stained with crystal violet.

RNA extraction and real-time quantitative PCR. Total RNA was extracted from the tissue samples using TRIzol. Then, total RNA (50 ng) was reverse-transcribed with miR-Let-7f stem-loop RT primers or with the U6 RT primers using a ReverTra Ace qPCR RT kit to generate cDNA. Real-time PCR was performed using a SYBR Premix Ex Taq ${ }^{\mathrm{TM}}$ kit with miR-Let-7f or U6 PCR primers. The reactions were performed using a LightCycler 2.0 instrument. U6 expression was used as the endogenous control. The absolute expression levels were calculated as concentration ratios using a Roche LightCycler $^{\circledR} 2.0$ system.

Immunohistochemistry staining. Paraffin-embedded human glioma tissue samples were sectioned and dewaxed. Endogenous peroxidase activity was quenched by incubating the slides in methanol containing $3 \%$ hydrogen peroxide for $30 \mathrm{~min}$, after which the sections were incubated for $2 \mathrm{~h}$ at room temperature with normal goat serum and subsequently incubated at $4^{\circ} \mathrm{C}$ overnight with primary antibody (1:300 CD34; Abcam). Next, the sections were incubated with horseradish peroxidase-linked second antibody, followed by reaction with diaminobenzidine and counterstaining with PAS staining kit and then Mayer's hematoxylin.

VM analysis. VM formation was evaluated using a commercial Matrigel matrix (BD Biosciences, France). A172 glioma cells were digested and resuspended at $5 \mathrm{x}^{4}$ cells $/ \mathrm{ml}$ in DMEM containing $1 \%$ FBS. Wells of 96 -well tissue culture plates were coated with Matrigel (50 $\mu \mathrm{l} /$ well; BD Biosciences) which was allowed to polymerase at $37^{\circ} \mathrm{C}$ for $30 \mathrm{~min}$. The glioma cell suspension was then plated at $100 \mu \mathrm{l} /$ well onto the surface of Matrigel and incubated at $37^{\circ} \mathrm{C}$. Cells were photographed using an Olympus inverted microscope.

Statistical analysis. All experiments were performed three times. The statistical analysis and experimental graphs were generated using SPSS 17.0 and GraphPad Prism software. Descriptive statistics including the means \pm SD, Mann-Whitney test, Kaplan-Meier plots, log-rank tests were used to analyze the significant differences. $\mathrm{p}<0.05$ and $\mathrm{p}<0.01$ were considered to indicate a statistically significant result.

\section{Results}

VM positively correlates with the WHO grades of human glioma tissues. To examine whether the VM correlates with 
Table I. Demographic parameters of patients participating in the present study.

\begin{tabular}{|c|c|c|}
\hline Characteristics & No. of patients & $\mathrm{N}(\%)$ \\
\hline \multicolumn{3}{|l|}{ Assessable } \\
\hline Glioma & 45 & 95.74 \\
\hline Normal brain tissues & 2 & 4.26 \\
\hline \multicolumn{3}{|l|}{ Gender } \\
\hline Male & 27 & 57.45 \\
\hline Female & 20 & 42.55 \\
\hline \multicolumn{3}{|l|}{ Age (years) } \\
\hline Median (range) & $42.6(4-69)$ & \\
\hline \multicolumn{3}{|l|}{ Pathological type } \\
\hline Astrocytoma & 6 & 12.77 \\
\hline Anaplastic astrocytoma & 10 & 21.28 \\
\hline Pilocytic astrocytoma & 2 & 4.26 \\
\hline Oligodendroglioma & 4 & 8.51 \\
\hline Anaplastic oligodendroglioma & 3 & 6.38 \\
\hline Glioblastoma & 20 & 42.56 \\
\hline Normal brain tissues & 2 & 4.26 \\
\hline \multicolumn{3}{|l|}{ WHO tumor grade at diagnosis } \\
\hline $\mathrm{I}$ & 4 & 8.89 \\
\hline II & 8 & 17.78 \\
\hline III & 13 & 28.89 \\
\hline IV & 20 & 44.44 \\
\hline
\end{tabular}

WHO, World Health Organization.

the WHO grades of human glioma, we assessed the VM in 45 human glioma specimens with different grades and 2 normal brain tissues by immunochemical staining (Table I). As shown in Fig. 1A, the VM was not found in the normal brain tissues and WHO grade I astrocytoma tissues, except pilocytic astrocytoma as its vascular hyperplasia. We also found that the VM rarely existed in WHO grade II glioma tissues (Figs. 1B and 2B). However, VM was very common in high-grade (WHO III and IV) gliomas, particularly in GBM (Fig. 1C and D). Accordingly, the VM-positive rate of human glioma was observably associated with its WHO grades (Fig. 2B).

$V M$ indicates a poor prognosis of glioma patients and negatively correlates with miR-Let-7f expression levels. To analyze whether VM in clinical samples associated with the clinical survival information of the 45 patients, Kaplan-Meier estimates were used in the present study. As expected, the subgroup of VM negative glioma patients presented a significantly prolonged postoperative survival time (Fig. 2A). Since our previous research found miR-Let-7f was identified as a potent anti-glioma miRNA (23), we hypothesized that miR-Let-7f was probably involved in the regulation process of VM. To assess the relationship between miR-Let-7f and VM, we verified the levels of miR-Let-7f in these 45 human glioma specimens by quantitative real-time PCR. The results showed that miR-Let-7f expression levels were negatively correlated with VM (Fig. 2C). These data were particularly strong for the VM suppressive effect of miR-Let-7f in glioma. However, its underlying mechanism of anti-VM effects remains unknown.

miR-Let-7f inhibits the migratory and VM forming capacities of human glioma cells. Migration is known to play an important role in tumor VM, and in an earlier study we showed that miR-Let-7f regulated the glioma cell migration (23). To determine whether miR-Let-7f could affect VM, we used the miR-Let-7f inhibitor and mimics. miRNA inhibitors are synthetic, 2'-O-methyl-modified, single-stranded molecules that interfere with miRNA function by sequestering them via irreversible binding $(24,25)$. Mimics are synthetic, double-stranded, modified RNA molecules that imitate the functions of endogenous miRNAs (26). As a proof of principle, the transient transfection of $80 \mathrm{nM}$ inhibitor or mimics did not affect glioma cell viability (Fig. 2D). In addition, we observed a significant pro-migratory effects of the miR-Let-7f inhibitor and anti-migratory effects of its mimics in wound healing and Transwell assays of A172 cells (Fig. 3A and B) in agreement with our findings previously reported. Furthermore, VM tube formation assay results confirmed that miR-Let-7f knockdown significantly promoted the VM formation and overexpression of miR-Let-7f completely blocked it (Fig. 3C). It suggests that miR-Let-7f may hinder the VM forming capacity of glioma cells by anti-migratory effects.

Overexpression of miR-Let-7f paralyses hypoxia-induced $V M$ formation. Several studies have demonstrated the hypoxia-induced angiogenesis and VM, and the pro-migratory effects of hypoxia. In this regard, we supposed that miR-Let- $7 \mathrm{f}$ was involved in the regulation of hypoxia-induced VM formation. After 6 h hypoxia treatment, A172 glioma cells revealed an excessively enhanced VM formation (Fig. 4B, upper).

To understand the mechanisms implicated in the VM suppressive role under hypoxia condition of miR-Let- $7 \mathrm{f}$, we investigated the time-dependent effects of miR-Let- $7 \mathrm{f}$ knockdown and overexpression in A172 glioma cells. First, we observed a significant VM suppression effect of miR-Let-7f mimics, particularly even under hypoxic conditions in A172 cells. While miR-Let-7f inhibitor significantly promoted A172 glioma cell VM under normoxic and hypoxia conditions (Fig. 4). Notably, the VM had a dynamic process with increased observation time. The undisturbed VM formation initiated at $4 \mathrm{~h}$, peaked at $6 \mathrm{~h}$ and vanished after $12 \mathrm{~h}$ in normoxia control A172 cells. In addition, miR-Let-7f inhibitor further enhanced the VM structures, particularly revealing the effect of hypoxia. In contrast, miR-Let-7f mimics completely blocked the VM formation throughout the experiment whether with hypoxia or not.

Taken together, our results clearly demonstrated that miR-Let-7f knockdown markedly promoted the VM forming capacity of human glioma cells and aggravate the hypoxia-induced VM promoting effects. While miR-Let-7f overexpression antagonized the VM formation, particularly the hypoxia-induced VM on human glioma cells.

miR-Let-7f antagonizes the hypoxia-induced VM promoting effects by targeting POSTN. As we previously reported, 


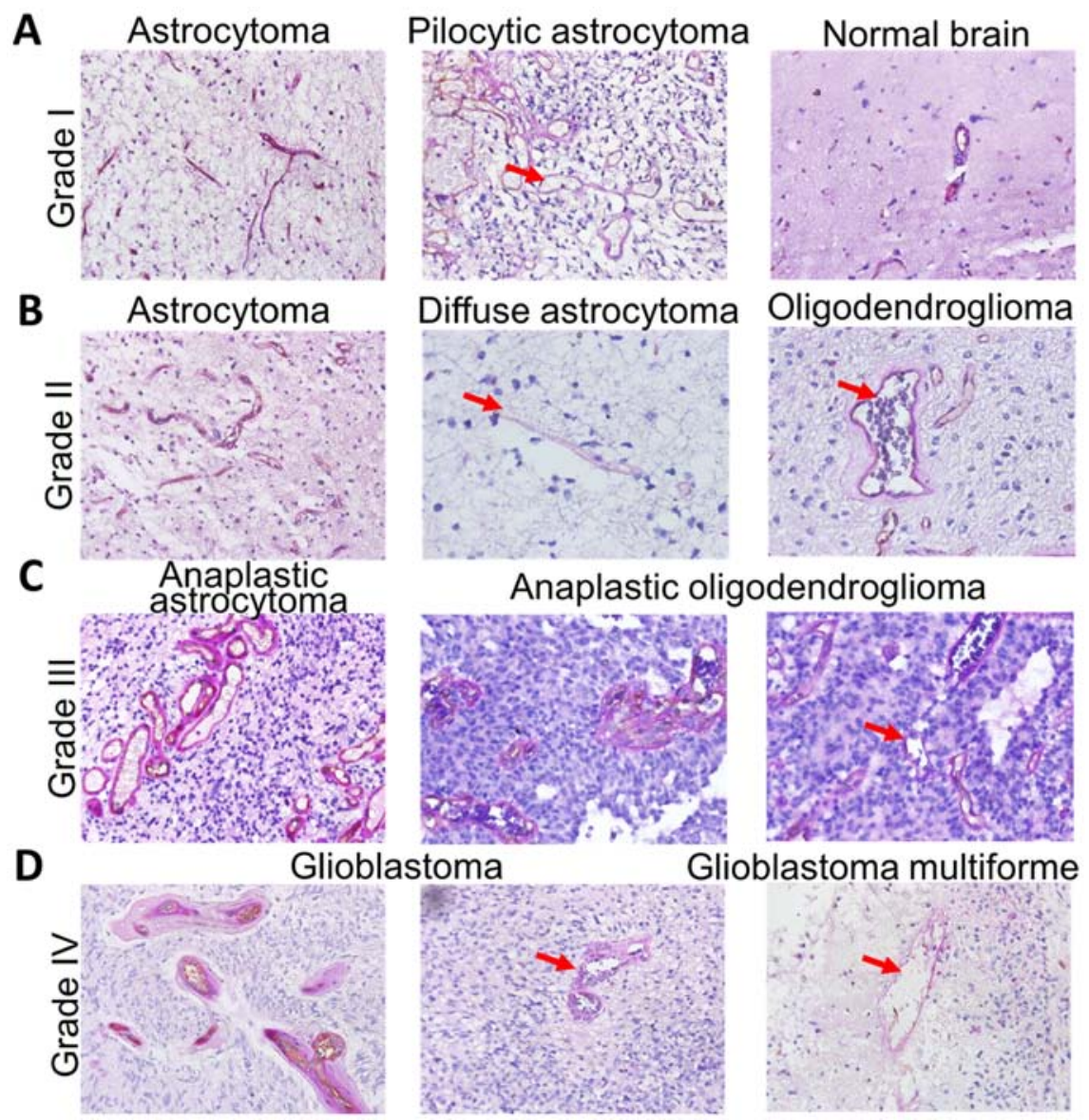

Figure 1. Vasculogenic mimicry in the human glioma tissues. (A) CD34-PAS immunochemical staining of WHO grade I glioma and normal brain. (B) The vasculogenic mimicry in WHO grade II glioma tissues (red arrow). (C) The vasculogenic mimicry in WHO grade III glioma tissues (red arrow). (D) The vasculogenic mimicry in WHO grade IV glioma tissues (red arrows).
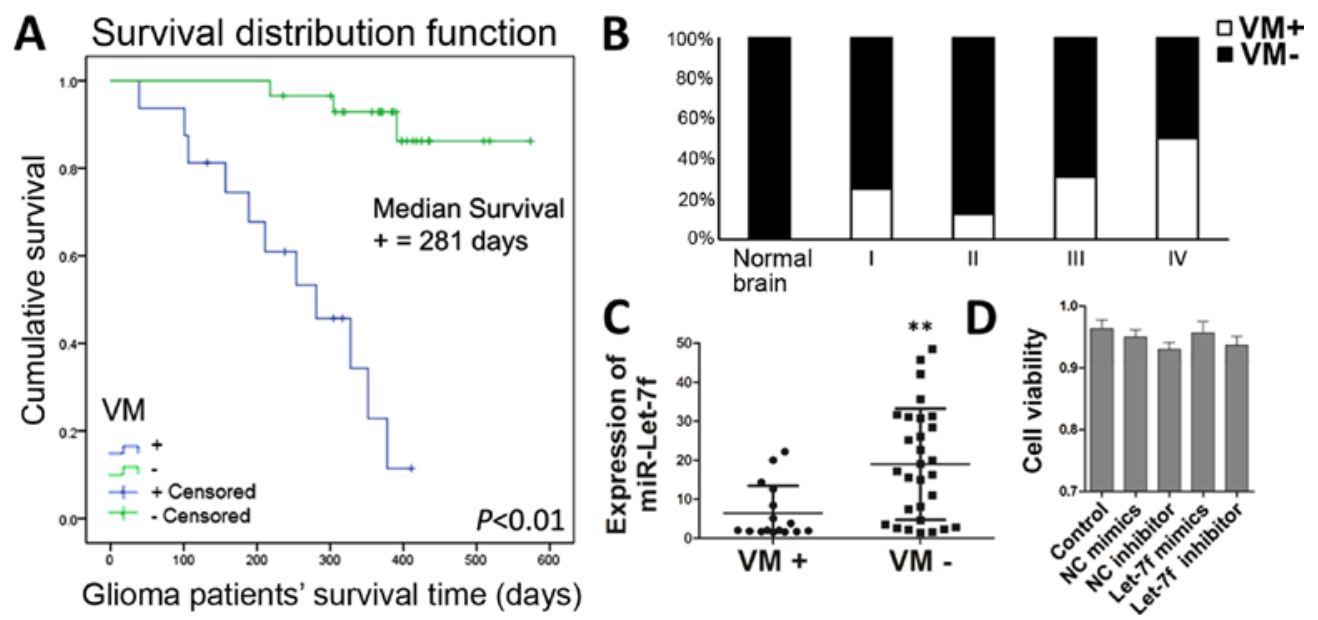

Figure 2. Vasculogenic mimicry positively correlates with the human glioma WHO grades, and VM functions as a prognostic indicator which negatively correlates with miR-Let-7f expression levels in human glioma tissues. (A) Prognostic significance of VM positive rate in glioma patients. According to the existence of VM, the Kaplan-Meier survival curves of glioma patients showed that VM correlated with poor prognosis. The Log Rank (Mantel-Cox) significance is $\mathrm{p}<0.01$. (B) The vasculogenic mimicry positive rate of human glioma was associated with WHO grades. (C) The miR-Let-7f expression levels in clinical samples from surgically removed glioma tissues of 45 patients (16 gliomas with VM and 29 gliomas without VM) were tested by quantitative real-time PCR. ${ }^{* *} \mathrm{p}=0.002$ by Mann-Whitney test. (D) Cell viability assay of A172 cells transfected with the miR-Let-7f mimics and inhibitor.

miR-Let-7f inhibited glioma migratory ability by directly targeting POSTN (23). It suggested that miR-Let-7f may block the VM forming capacity of glioma cells by regulating the POSTN-dependent migration. To investigate whether miR-
Let-7f and POSTN are linked, we utilized a POSTN siRNA. siRNA transfected A172 glioma cells almost lost the migratory and VM forming ability as miR-Let-7f overexpressed cells (Fig. 5, middle), and the VM promoting effect of miR- 


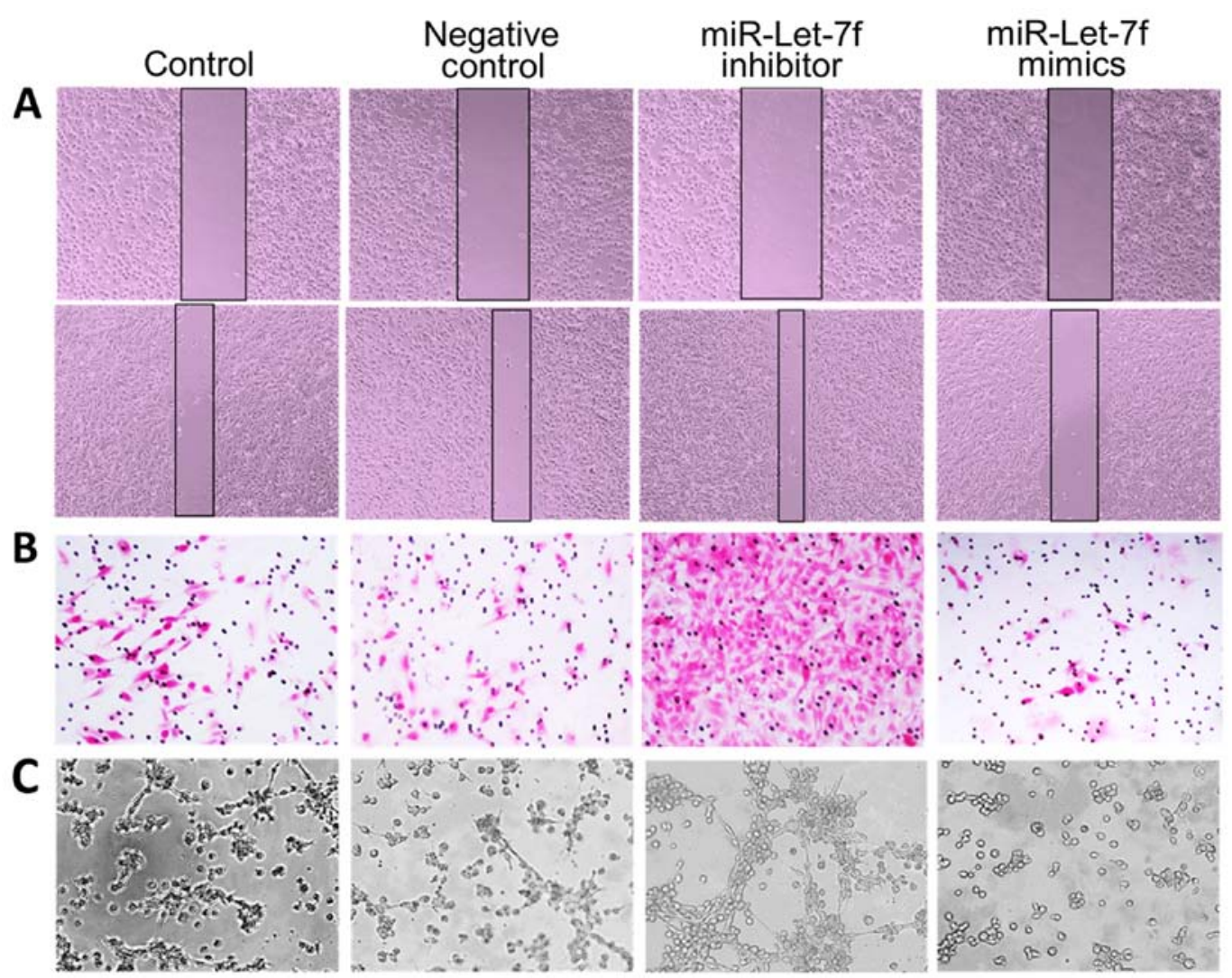

Figure 3. miR-Let-7f inhibits the migratory and VM forming capacities of human glioma cells. The pro-migratory effect of the miR-Let-7f inhibitor and anti-migratory effect of the miR-Let-7f mimics was examined by wound healing assay (A) Transwell migration assays (B) on A172 cell migration. At $48 \mathrm{~h}$ after transfection, a cell suspension was added to the upper chamber of an uncoated Transwell membrane insert, and the lower chamber was filled with media The cells were cultured under normoxia conditions for $12 \mathrm{~h}$. Then, migratory cells were stained, and the average number of cells was counted in triplicate. (C) Effect of miR-Let-7f mimic and inhibitor transfection on A172 cell VM formation under normoxic conditions. After the cells were transfected and incubated, they were transferred into wells of 96 -well tissue culture plates coated with Matrigel in the Materials and methods.

\section{A} $4 \mathrm{~h}$
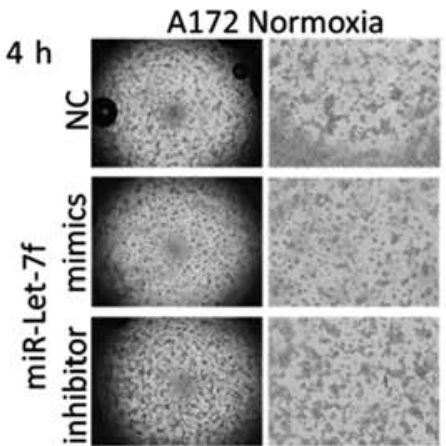

C
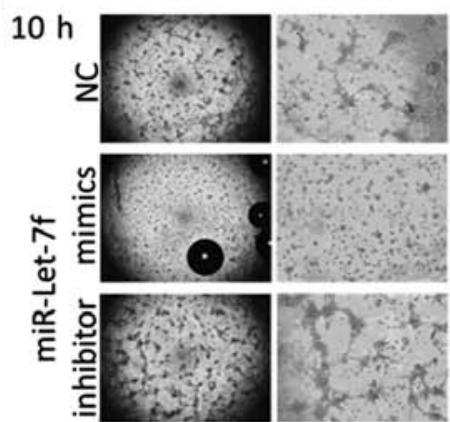

A172 Hypoxia
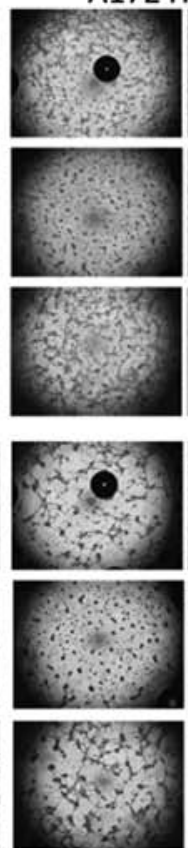
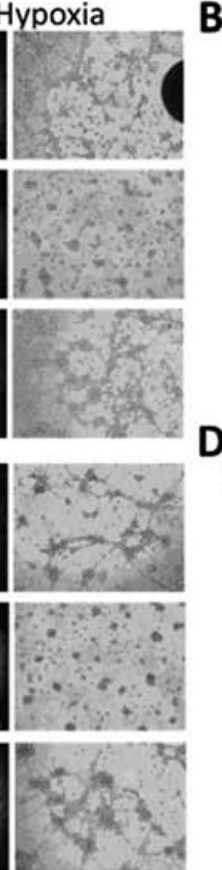

B $6 \mathrm{~h}$
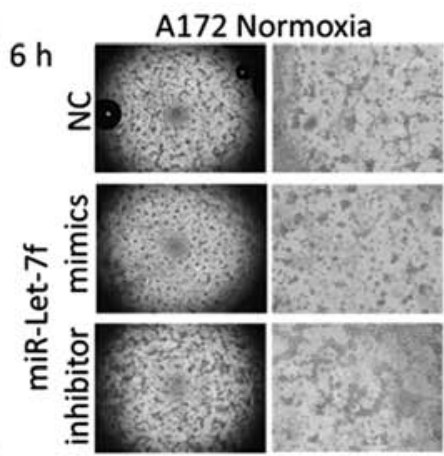

$12 \mathrm{~h}$
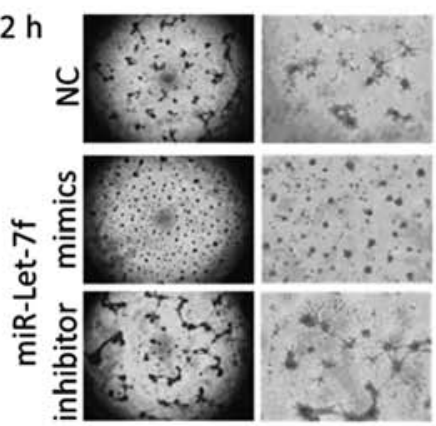

A172 Hypoxia
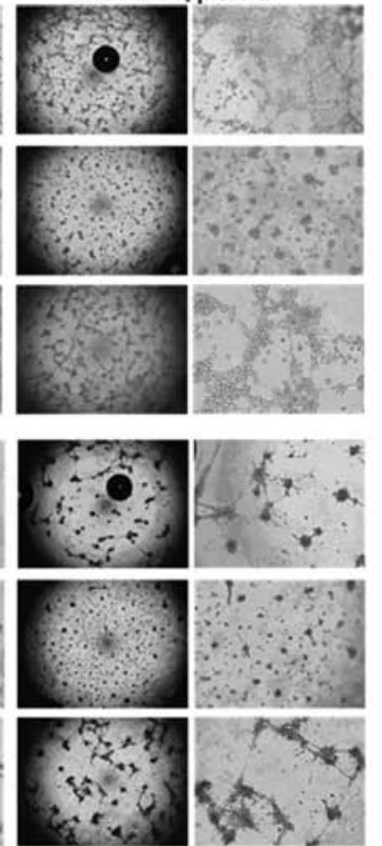

Figure 4. Overexpression of miR-Let-7f paralyses hypoxia-induced VM formation in human A172 glioma cells. The VM suppression effect of miR-Let-7f mimics, particularly even under hypoxic conditions in A172 cells. While miR-Let-7f inhibitor significantly promoted A172 glioma cell VM under normoxic and hypoxia conditions. The undisturbed VM formation was initiated at $4 \mathrm{~h}$, peaked at $6 \mathrm{~h}$ and vanished after $12 \mathrm{~h}$ in normoxia control A172 cells. In addition, miR-Let-7f inhibitor further enhanced the VM structures, particularly revealing the effect of hypoxia. In contrast, miR-Let-7f mimics completely blocked the VM formation throughout the experiment whether with hypoxia or not. 


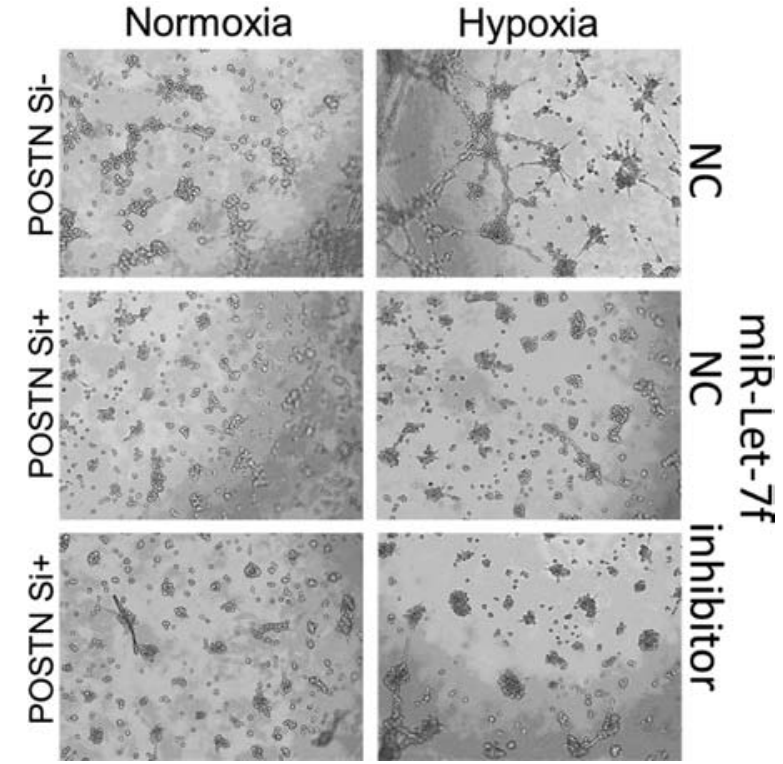

Figure 5. Specific inhibition of POSTN by siRNA blocks the VM promoting effect of the miR-Let-7f inhibitor and exerts a synergistic effect with the miRLet-7f mimic. POSTN siRNA transfection of A172 glioma cells resulted in significant inhibition of VM (upper and middle). The miR-Let-7f and POSTN siRNA co-transfected A172 cells showed that the VM promoting effect of miR-Let-7f inhibitor was totally blocked (lower). In addition, the hypoxia effect was also blocked by POSTN-specific inhibition (right).

Let-7f inhibitor was totally blocked (Fig. 5, lower). In addition, the hypoxia effect was also blocked by POSTN knockdown (Fig. 5, right). Hence we could confirm that miR-Let-7f antagonizes the hypoxia-induced VM promoting effects by targeting POSTN.

\section{Discussion}

There is growing interest in exploring the tumor-suppressive miRNAs, and accumulating evidence demonstrates their potential of prognostic and antitumor therapeutic prospects $(27,28)$. Several recent studies have confirmed numerous highly expressed miRNA predictors $(13,29-31)$ for glioma prognosis. Moreover, an increasing number of tumorsuppressive miRNAs has also been discovered (32). In the present study, we identified a novel antitumor mechanism of miR-Let-7f by its anti-vasculogenic mimicry (VM) effect in human glioma, and its possible regulatory target in malignant glioma.

Cells undergo a variety of biological responses when subjected to ischemic and hypoxic conditions, including the activation of signaling pathways that regulate proliferation, angiogenesis, metastasis and apoptosis. Tumor cells have adapted these pathways to survive and even grow under ischemic and hypoxic conditions. Tumor hypoxia is associated with poor prognosis and resistance to antitumor therapy (33). Additionally, hypoxia-induced angiogenesis is also a common feature in solid tumors due to their overwhelming progression and relatively inadequate blood supply. Cancer stem cells (CSCs) were identified as a VM-initiating cells in many types of cancer (34). The detailed VM phenomenon in non-GSC glioma cells has been reported (35), but its mechanisms are still unclear. Vascularization is crucial for the growth of hypoxic tumors (36), and VM as a new vascular type described as the non-endothelium-dependent vessels in gliomas (37), represents an important new tumor survival mechanism. VM may contribute to the failure of current anti-angiogenic therapy (38), and the exact mechanism still requires further study.

Then, we first investigated the effect of miR-Let-7f as a new potential tumor suppressor based on its significant VM inhibition in hypoxic glioma cells. However, the pathways implicated in the tumor suppressive role of miR-Let-7f are poorly defined. Our results suggest that miR-Let-7f may suppress VM by inhibiting the migration capacity of glioma cells. Based on our results and previous reports, we validated the role of potential target POSTN in VM using the specific siRNA. This inhibitor prevented the VM promoting effect of the miR-Let-7f inhibitor as expected. These results demonstrated that POSTN is a direct target of miR-Let-7f in VM forming regulation process.

In summary, the mechanism by which miR-Let-7f functions is summarized as follows. First, miR-Let-7f downregulates POSTN directly and inhibits POSTN-mediated migration. Consequently, this decreased cell motility significantly induced the glioma cell VM forming failure. Ultimately, reduced blood supply improved the prognosis of glioma patients. However, a limitation of the present study is the small number of samples, and the involvement of other key invasion-associated proteins such as Rac1, Cdc42 (39) or MMPs (40) were not investigated. Therefore, additional studies will be required to substantiate this mechanism.

\section{Acknowledgements}

The present study was supported by grants from the National Natural Science Foundation of China (nos. 81101594, 81372719, 81172403, 81402077, 81571284 and 91542115). We thank the senior editor of American Journal Experts for linguistic advice.

\section{References}

1. Ohgaki H: Epidemiology of brain tumors. Methods Mol Biol 472: 323-342, 2009

2. Ostrom QT, Gittleman H, Stetson L, Virk SM and BarnholtzSloan JS: Epidemiology of gliomas. Cancer Treat Res 163: 1-14, 2015.

3. McNamara MG and Mason WP: Antiangiogenic therapies in glioblastoma multiforme. Expert Rev Anticancer Ther 12: 643-654, 2012.

4. Vaupel P: Hypoxia and aggressive tumor phenotype: Implications for therapy and prognosis. Oncologist 13 (Suppl 3): S21-S26, 2008.

5. Mongiardi MP: Angiogenesis and hypoxia in glioblastoma: A focus on cancer stem cells. CNS Neurol Disord Drug Targets 11: 878-883, 2012.

6. Ji RC: Hypoxia and lymphangiogenesis in tumor microenvironment and metastasis. Cancer Lett 346: 6-16, 2014.

7. Seftor RE, Hess AR, Seftor EA, Kirschmann DA, Hardy KM, Margaryan NV and Hendrix MJ: Tumor cell vasculogenic mimicry: From controversy to therapeutic promise. Am J Pathol 181: 1115-1125, 2012.

8. Qiao L, Liang N, Zhang J, Xie J, Liu F, Xu D, Yu X and Tian Y: Advanced research on vasculogenic mimicry in cancer. J Cell Mol Med 19: 315-326, 2015.

9. Dong J, Zhao Y, Huang Q, Fei X, Diao Y, Shen Y, Xiao H, Zhang T, Lan Q and Gu X: Glioma stem/progenitor cells contribute to neovascularization via transdifferentiation. Stem Cell Rev 7: 141-152, 2011. 
10. Liu T, Sun B, Zhao X, Li Y, Gu Q, Dong X and Liu F: OCT4 expression and vasculogenic mimicry formation positively correlate with poor prognosis in human breast cancer. Int $\mathbf{J} \mathrm{Mol}$ Sci 15: 19634-19649, 2014

11. Bartel DP: MicroRNAs: Genomics, biogenesis, mechanism, and function. Cell 116: 281-297, 2004.

12. Filipowicz W, Bhattacharyya SN and Sonenberg N: Mechanisms of post-transcriptional regulation by microRNAs: Are the answers in sight? Nat Rev Genet 9: 102-114, 2008.

13. Zhang R, Pang B, Xin T, Guo H, Xing Y, Xu S, Feng B, Liu B and Pang Q: Plasma miR-221/222 family as novel descriptive and prognostic biomarkers for glioma. Mol Neurobiol: Jan 31, 2015 (Epub ahead of print)

14. Dews M, Homayouni A, Yu D, Murphy D, Sevignani C, WentzelE, Furth EE, Lee WM, Enders GH, Mendell JT, et al: Augmentation of tumor angiogenesis by a Myc-activated microRNA cluster. Nat Genet 38: 1060-1065, 2006.

15. Ma L, Young J, Prabhala H, Pan E, Mestdagh P, Muth D, Teruya-Feldstein J, Reinhardt F, Onder TT, Valastyan S, et al: miR-9, a MYC/MYCN-activated microRNA, regulates E-cadherin and cancer metastasis. Nat Cell Biol 12: 247-256, 2010.

16. Zhao Z, Tan X, Zhao A, Zhu L, Yin B, Yuan J, Qiang B and Peng X: microRNA-214-mediated UBC9 expression in glioma. BMB Rep 45: 641-646, 2012

17. Li X, Ling N, Bai Y, Dong W, Hui GZ, Liu D, Zhao J and Hu J: MiR-16-1 plays a role in reducing migration and invasion of glioma cells. Anat Rec 296: 427-432, 2013.

18. Ying Z, Li Y, Wu J, Zhu X, Yang Y, Tian H, Li W, Hu B, Cheng SY and Li M: Loss of miR-204 expression enhances glioma migration and stem cell-like phenotype. Cancer Res 73: 990-999, 2013

19. Lee HK, Bier A, Cazacu S, Finniss S, Xiang C, Twito $H$, Poisson LM, Mikkelsen T, Slavin S, Jacoby E, et al MicroRNA-145 is downregulated in glial tumors and regulates glioma cell migration by targeting connective tissue growth factor. PLoS One 8: e54652, 2013.

20. Gao R, Cai C, Gan J, Yang X, Shuang Z, Liu M, Li S and Tang H: miR-1236 down-regulates alpha-fetoprotein, thus causing PTEN accumulation, which inhibits the PI3K/Akt pathway and malignant phenotype in hepatoma cells. Oncotarget 6: 6014-6028, 2015.

21. Song Y, Mu L, Han X, Li Q, Dong B, Li H and Liu X: MicroRNA-9 inhibits vasculogenic mimicry of glioma cell lines by suppressing Stathmin expression. J Neurooncol 115: 381-390, 2013.

22. Wu N, Zhao X, Liu M, Liu H, Yao W, Zhang Y, Cao S and Lin X: Role of microRNA-26b in glioma development and its mediated regulation on EphA2. PLoS One 6: e16264, 2011.

23. Yan S,Han X, Xue H,Zhang P,Guo X,Li T, GuoX, Yuan G, Deng L and Li G: Let-7f inhibits glioma cell proliferation, migration, and invasion by targeting periostin. J Cell Biochem 116: 1680-1692, 2015.

24. Meister G, Landthaler M, Dorsett Y and Tuschl T: Sequencespecific inhibition of microRNA- and siRNA-induced RNA silencing. RNA 10: 544-550, 2004.
25. Hutvágner G, Simard MJ, Mello CC and Zamore PD: Sequencespecific inhibition of small RNA function. PLoS Biol 2: E98, 2004.

26. Kim DH and Rossi JJ: Strategies for silencing human disease using RNA interference. Nat Rev Genet 8: 173-184, 2007.

27. Yan W, Li R, Liu Y, Yang P, Wang Z, Zhang C, Bao Z, Zhang W, You $\mathrm{Y}$ and Jiang T: MicroRNA expression patterns in the malignant progression of gliomas and a 5-microRNA signature for prognosis. Oncotarget 5: 12908-12915, 2014.

28. Auffinger B, Thaci B, Ahmed A, Ulasov I and Lesniak MS: MicroRNA targeting as a therapeutic strategy against glioma. Curr Mol Med 13: 535-542, 2013.

29. Yang CH, Yue J, Pfeffer SR, Fan M, Paulus E, Hosni-Ahmed A, Sims M, Qayyum S, Davidoff AM, Handorf CR, et al: MicroRNA-21 promotes glioblastoma tumorigenesis by down-regulating insulin-like growth factor-binding protein-3 (IGFBP3). J Biol Chem 289: 25079-25087, 2014.

30. Barbano R, Palumbo O, Pasculli B, Galasso M, Volinia S, D'Angelo V, Icolaro N, Coco M, Dimitri L, Graziano P, et al: A miRNA signature for defining aggressive phenotype and prognosis in gliomas. PLoS One 9: e108950, 2014.

31. Lai NS, Wu DG, Fang XG, Lin YC, Chen SS, Li ZB and Xu SS: Serum microRNA-210 as a potential noninvasive biomarker for the diagnosis and prognosis of glioma. Br J Cancer 112: 1241-1246, 2015.

32. Que T, Song Y, Liu Z, Zheng S, Long H, Li Z, Liu Y, Wang G, Liu Y, Zhou J, et al: Decreased miRNA-637 is an unfavorable prognosis marker and promotes glioma cell growth, migration and invasion via direct targeting Akt1. Oncogene 34: 4952-4963, 2015.

33. Harris AL: Hypoxia - a key regulatory factor in tumour growth. Nat Rev Cancer 2: 38-47, 2002.

34. Fan YL, Zheng M, Tang YL and Liang XH: A new perspective of vasculogenic mimicry: EMT and cancer stem cells (Review). Oncol Lett 6: 1174-1180, 2013.

35. Smith SJ, Ward JH, Tan C, Grundy RG and Rahman R: Endothelial-like malignant glioma cells in dynamic three dimensional culture identifies a role for VEGF and FGFR in a tumor-derived angiogenic response. Oncotarget 6: 22191-22205, 2015.

36. Jhaveri N, Chen TC and Hofman FM: Tumor vasculature and glioma stem cells: Contributions to glioma progression. Cancer Lett: Dec 16, 2014 (Epub ahead of print). pii: S0304-3835(14)00783-6. doi: 10.1016/j.canlet.2014.12.028.

37. Yue WY and Chen ZP: Does vasculogenic mimicry exist in astrocytoma? J Histochem Cytochem 53: 997-1002, 2005.

38. Mao JM, Liu J, Guo G, Mao XG and Li CX: Glioblastoma vasculogenic mimicry: Signaling pathways progression and potential anti-angiogenesis targets. Biomark Res 3, 2015.

39. Cardama GA, Gonzalez N, Ciarlantini M, Gandolfi Donadío L, Comin MJ, Alonso DF, Menna PL and Gomez DE: Proapoptotic and antiinvasive activity of Rac1 small molecule inhibitors on malignant glioma cells. Onco Targets Ther 7: 2021-2033, 2014.

40. Könnecke $\mathrm{H}$ and Bechmann I: The role of microglia and matrix metalloproteinases involvement in neuroinflammation and gliomas. Clin Dev Immunol 2013: 914104, 2013. 